Article

\title{
Preoperative Serum Calcitonin and Its Correlation with Extent of Lymph Node Metastasis in Medullary Thyroid Carcinoma
}

\author{
Hyunju Park ${ }^{1}$, Jun Park ${ }^{1}$, Min Sun Choi ${ }^{1}$, Jinyoung Kim ${ }^{1} \oplus$, Hosu Kim ${ }^{2}{ }^{\circledR}$, Jung Hee Shin ${ }^{3}$, \\ Jung-Han Kim ${ }^{4}$, Jee Soo Kim ${ }^{4} \oplus$, Sun Wook Kim ${ }^{1}$, Jae Hoon Chung ${ }^{1}{ }^{\circledR}$ and Tae Hyuk Kim ${ }^{1, *}{ }^{1}$ \\ 1 Division of Endocrinology \& Metabolism, Department of Medicine, Thyroid Center, \\ Samsung Medical Center, Sungkyunkwan University School of Medicine, Seoul 06351, Korea; \\ hj1006.park@samsung.com (H.P.); jun113.park@samsung.com (J.P.); minsun1.choi@samsung.com (M.S.C.); \\ j513.kim@samsung.com (J.K.); sunwooksmc.kim@samsung.com (S.W.K.); thyroid@skku.edu (J.H.C.) \\ 2 Division of Endocrinology, Department of Medicine, Gyeongsang National University Changwon Hospital, \\ Gyeongsang National University College of Medicine, Changwon 51472, Korea; narulake@naver.com \\ 3 Department of Radiology, Samsung Medical Center, Sungkyunkwan University School of Medicine, \\ Seoul 06351, Korea; helena35.shin@samsung.com \\ 4 Division of Breast and Endocrine Surgery, Department of Surgery, Samsung Medical Center, \\ Sungkyunkwan University School of Medicine, Seoul 06351, Korea; jinnee.kim@samsung.com (J.-H.K.); \\ js0507.kim@samsung.com (J.S.K.) \\ * Correspondence: taehyukmd.kim@samsung.com; Tel.: +82-2-3410-6049
}

Received: 7 September 2020; Accepted: 7 October 2020; Published: 9 October 2020

Simple Summary: Surgery is the only curative treatment for medullary thyroid carcinoma (MTC), but the initial surgical extent is still controversial. We examined whether the preoperative serum calcitonin level reflects the extent of lymph node metastasis (LNM), and therefore might be used to predict the optimal initial surgical extent for MTC. Furthermore, positive and negative likelihood ratios for preoperative serum calcitonin were calculated for calcitonin concentration categories, revealing that serum calcitonin levels can be of diagnostic value and might be applicable to surgical decision-making.

\begin{abstract}
The optimal initial surgical extent for medullary thyroid carcinoma (MTC) remains controversial. Previous studies on serum calcitonin are limited to reporting the calcitonin threshold according to anatomical disease burden. Here, we evaluated whether preoperative calcitonin levels can be used to predict optimal surgical extent. We retrospectively reviewed the 170 patients with MTC at a tertiary Korean hospital from 1994 to 2019. We extracted data on preoperative calcitonin level, primary tumor size and the number and location of lymph node metastases (LNMs). To evaluate disease extent, we divided the patients into five groups: no LNM, central LNM, ipsilateral lateral LNM, contralateral lateral LNM, and distant metastasis. We calculated the positive and negative likelihood ratios (LRs) for multiple categories of preoperative calcitonin levels. Preoperative calcitonin level positively correlated with primary tumor size (rho $=0.744, p<0.001)$ and LNM number ( rho $=0.537$, $p<0.001$ ). Preoperative calcitonin thresholds of 20,200 , and $500 \mathrm{pg} / \mathrm{mL}$ were associated with the presence of ipsilateral lateral LNM, contralateral lateral LNM, and distant metastasis, respectively. The negative LRs were 0.1 at a preoperative calcitonin cut-off of $100 \mathrm{pg} / \mathrm{mL}$ in the central LNM, 0.18 at a cut-off of $300 \mathrm{pg} / \mathrm{mL}$ in the ipsilateral lateral LNM, and 0 at a cut-off of $300 \mathrm{pg} / \mathrm{mL}$ in the contralateral lateral LNM. The preoperative calcitonin level correlates with disease extent and has diagnostic value for predicting LNM extent. Our results suggest that the preoperative calcitonin level can be used to determine optimal initial surgical extent.
\end{abstract}

Keywords: medullary thyroid carcinoma; calcitonin; lymph nodes; lymph node excision; clinical decision-making 


\section{Introduction}

Medullary thyroid carcinoma (MTC) accounts for 1-2\% of all thyroid carcinomas in the United States, and $0.4-2.2 \%$ of all thyroid carcinomas in Korea [1-3]. The 10-year survival rate for the MTC is $75-85 \%$, whereas the 10 -year survival is about $90 \%$ for papillary thyroid carcinoma [4-6]. It occurs as both a sporadic and a familial disease, and in families, MTC can occur alone or in combination with multiple endocrine neoplasia type 2A (MEN-2A) or MEN-2B [2].

MTC arises from thyroid parafollicular cells (C cells), which secrete several hormones, including calcitonin. Calcitonin is a 32-amino acid monomeric peptide that is used as a highly sensitive and specific tumor marker for MTC $[7,8]$. The current American Thyroid Association (ATA) guidelines recommend measuring the serum calcitonin level when patients are diagnosed with histological MTC [9]. Additionally, serum calcitonin levels can be used in differential diagnosis, prognostic assessments, and evaluations of treatment response [10-14].

The standard initial treatment for MTC is surgery, and surgery is the only curative treatment option $[9,15,16]$. However, the extent of initial surgery for MTC is controversial because of the possibility of complications and its unknown benefits. The ATA guidelines recommend total thyroidectomy and the central compartment dissection of cervical lymph nodes, regardless of the presence of abnormal lymph nodes as determined by ultrasound [9]. By contrast, despite the general agreement that the therapeutic procedure for both sporadic and familial MTC is total thyroidectomy with central lymph node dissection, the National Comprehensive Center Network (NCCN) guidelines suggest that central neck dissection may not be necessary for MTCs $\leq 1 \mathrm{~cm}$ [15]. Based on a previous study reporting that ipsilateral and contralateral lateral lymph node metastasis (LNM) is associated with basal serum calcitonin levels exceeding 20 and $200 \mathrm{pg} / \mathrm{mL}$, respectively [17], the current guidelines suggest that lateral lymph node dissection may be considered as a result of the serum calcitonin level and the results of ultrasound scans $[9,16]$. However, previous studies have reported threshold calcitonin levels depending only on the presence of LNM $[12,17,18]$.

Here, we hypothesized that if the preoperative serum calcitonin level reflects the extent of LNM, the preoperative serum calcitonin level could also be a useful biomarker for tailoring the optimal initial surgical extent, particularly for lymph node dissection in the lateral neck compartment. Thus, we evaluated the correlation between preoperative serum calcitonin and the existence of LNM in MTC, and provide concrete stochastic information on the preoperative serum calcitonin level that could determine the optimal surgical extent.

\section{Results}

\subsection{Baseline Characteristics and Factors Associated with Elevations in Preoperative Serum Calcitonin}

The mean age of the 170 patients with MTC who had data on their preoperative serum calcitonin levels was $49.5 \pm 14.5$ years, and 111 patients $(65.3 \%)$ were female. The median primary tumor size was $1.5(0.7-2.5) \mathrm{cm}$, and the median preoperative serum calcitonin concentration was 401 (96.9-1398.5) pg/mL. The mean number of LNMs was 4.9 (8.6), and LNM was present in $148(87.1 \%)$ patients. Among the patients with LNM, $72(42.4 \%)$ patients, 62 patients $(36.5 \%)$ and 14 patients $(8.2 \%)$ had central LNM, ipsilateral lateral LNM, and contralateral lateral LNM, respectively. Eight patients $(4.7 \%)$ had initial distant metastasis. Among the 170 patients in this study, 62 were classified as having a node stage above $\mathrm{N} 1 \mathrm{~b}$. The group with a node stage below $\mathrm{N} 1 \mathrm{~b}$ had a higher proportion of females, a smaller primary tumor size, lower preoperative serum calcitonin levels and a lower number of LNMs compared to the group with a node stage above N1b (Table 1).

Because the preoperative serum calcitonin level was higher in the group with a node stage above $\mathrm{N} 1 \mathrm{~b}$, we performed linear regression analyses to identify the factors that correlated with the preoperative serum calcitonin level. The multivariate linear regression analysis showed that log-calcitonin was significantly associated with primary tumor size $(b=0.33$ and $p<0.001)$, node stage $\mathrm{N} 1 \mathrm{~b}(\mathrm{~b}=0.47$ and $p<0.001$ ), and positive extrathyroidal extension (ETE) $(b=0.26$ and $p=0.05)$ (Table 2). 
Table 1. Baseline clinicopathologic characteristics of the patients.

\begin{tabular}{|c|c|c|c|c|}
\hline Characteristic & $\begin{array}{l}\text { Number of Patients } \\
\quad(n=170)\end{array}$ & $\begin{array}{l}\text { Below N1b } \\
\quad(n=108)\end{array}$ & $\begin{array}{l}\text { Above N1b } \\
\quad(n=62)\end{array}$ & $p$ Value \\
\hline Age (mean \pm SD) & $49.5 \pm 14.5$ & $50.5 \pm 14.0$ & $47.7 \pm 15.4$ & 0.235 \\
\hline \multicolumn{5}{|l|}{$\operatorname{Sex}(\%)$} \\
\hline Female & 111 (65.3) & 78 (72.2) & $33(53.2)$ & \multirow{2}{*}{0.012} \\
\hline Male & $59(34.7)$ & $30(27.8)$ & $29(46.8)$ & \\
\hline \multicolumn{5}{|l|}{ Tumor type (\%) } \\
\hline Sporadic & $139(81.8)$ & $85(78.7)$ & $54(87.1)$ & \multirow{2}{*}{0.173} \\
\hline Hereditary (MEN-2A) & $31(18.2)$ & $23(21.3)$ & $9(12.9)$ & \\
\hline Primary tumor size (median, IQR) & $1.5(0.7-2.5)$ & $1.2(0.6-1.9)$ & $2.0(1.3-3.5)$ & $<0.001$ \\
\hline $\begin{array}{l}\text { Preoperative serum calcitonin } \\
\text { (pg/mL, median, IQR) }\end{array}$ & $\begin{array}{c}401.0 \\
(96.93-1398.5)\end{array}$ & $\begin{array}{c}162.5 \\
(40.8-587.0)\end{array}$ & $\begin{array}{c}1201.0 \\
(554.3-2827.0)\end{array}$ & $<0.001$ \\
\hline \multicolumn{5}{|l|}{ Extrathyroidal extension (\%) } \\
\hline Negative & $125(73.5)$ & $98(90.7)$ & $27(43.5)$ & \multirow{2}{*}{$<0.001$} \\
\hline Positive & $45(26.5)$ & $10(9.3)$ & $35(56.5)$ & \\
\hline Number of LNMs (mean \pm SD) & $4.9(8.6)$ & $0.3(1.0)$ & $12.8(10.1)$ & $<0.001$ \\
\hline \multicolumn{5}{|l|}{ Extent of LNM $(\%)$} \\
\hline Central LNM & $72 / 170(42.4)$ & 17/108 (15.7) & $55 / 62(88.7)$ & $<0.001$ \\
\hline Ipsilateral lateral LNM & $62 / 170(36.5)$ & 0 & $62 / 62(100)$ & $<0.001$ \\
\hline Contralateral lateral LNM & $14 / 170(8.2)$ & 0 & $14 / 62(22.6)$ & $<0.001$ \\
\hline Distant metastasis (\%) & $8 / 170(4.7)$ & 0 & $8 / 62(12.9)$ & $<0.001$ \\
\hline \multicolumn{5}{|l|}{ AJCC 8th stage (\%) } \\
\hline 1 & $68(40.0)$ & $68(63.0)$ & $0(0)$ & \multirow{6}{*}{$<0.001$} \\
\hline 2 & $23(13.5)$ & $23(21.3)$ & $0(0)$ & \\
\hline 3 & $17(10.0)$ & $17(15.7)$ & $0(0)$ & \\
\hline $4 a$ & $54(31.8)$ & $0(0)$ & $53(85.5)$ & \\
\hline $4 b$ & $0(0)$ & $0(0)$ & $1(1.6)$ & \\
\hline $4 c$ & $8(4.7)$ & 0 & $8(12.9)$ & \\
\hline
\end{tabular}

$\mathrm{N} 1 \mathrm{~b}=$ Presence of unilateral, bilateral, or contralateral lateral lymph node metastasis; $\mathrm{SD}=$ standard deviation; $\mathrm{IQR}=$ interquartile range; $\mathrm{MEN}-2 \mathrm{~A}$ = multiple endocrine neoplasia type $2 \mathrm{~A} ; \mathrm{LNM}=$ lymph node metastasis; $\mathrm{AJCC}=$ American Joint Committee on Cancer.

Table 2. Linear regression analysis of preoperative serum calcitonin ( $\mathrm{pg} / \mathrm{mL}$, log-transformed) and the indicated factors.

\begin{tabular}{ccccc}
\hline \multirow{2}{*}{ Characteristic } & \multicolumn{2}{c}{ Unadjusted } & \multicolumn{2}{c}{ Adjusted } \\
\cline { 2 - 5 } & $\mathbf{b} \pm \mathbf{S E}$ & $p$ Value & $\mathbf{b} \pm \mathbf{S E}$ & $p$ Value \\
\hline Sex (female) & $0.137 \pm 0.103$ & 0.188 & & \\
Age at diagnosis (years) & $0.006 \pm 0.004$ & 0.083 & & \\
Primary tumor size & $0.328 \pm 0.035$ & $<0.001$ & $0.327 \pm 0.035$ & $<0.001$ \\
Nodal metastasis above N1b & $0.529 \pm 0.121$ & $<0.001$ & $0.472 \pm 0.118$ & $<0.001$ \\
Positive extrathyroidal extension & $0.265 \pm 0.131$ & 0.045 & $0.257 \pm 0.131$ & 0.050 \\
Positive resection margin & $-0.057 \pm 0.457$ & 0.901 & & \\
Tumor type (sporadic/hereditary) & $0.239 \pm 0.134$ & 0.076 & & \\
\hline & SE $=$ standard error.
\end{tabular}

\subsection{Tumor Burden and Correlation with Preoperative Serum Calcitonin}

Next, we assessed the relationships among the preoperative serum calcitonin levels, primary tumor size, and number of LNMs (Table 3). Because the preoperative serum calcitonin levels of the patients did not show a linear increase, we classified the serum calcitonin levels into eight categories, whereby category 1 was the lowest concentration range and category 8 was the highest. We found statistically significant relationships between the eight groups of increasing preoperative serum calcitonin levels and primary tumor size (rho $=0.744, p<0.001$ ) as well as the number of LNMs (rho $=0.537, p<0.001$ ). 
Table 3. Tumor extent and cancer prognosis according to preoperative serum calcitonin levels.

\begin{tabular}{ccccccc}
\hline \multirow{2}{*}{$\begin{array}{c}\text { Calcitonin } \\
\text { Category }\end{array}$} & \multirow{2}{*}{$\begin{array}{c}\text { Preoperative Serum } \\
\text { Calcitonin }(\mathbf{p g} / \mathbf{m L})\end{array}$} & \multirow{2}{*}{$\begin{array}{c}\text { Number } \\
\text { of Patients }\end{array}$} & \multicolumn{2}{c}{ Primary Tumor Size } & \multicolumn{2}{c}{ Number of LNMs } \\
\cline { 5 - 7 } & & & Mean & $\mathbf{9 5 \%}$ CI & Mean & 95\% CI \\
\hline 1 & $1-20$ & 22 & 0.61 & $0.36-0.86$ & 0.32 & $0-0.82$ \\
2 & $20.1-100$ & 22 & 0.81 & $0.67-0.96$ & 0.41 & $0-1.26$ \\
3 & $100.1-200$ & 18 & 1.59 & $1.03-2.16$ & 1.61 & $0-3.56$ \\
4 & $200.1-500$ & 30 & 1.29 & $1.07-1.51$ & 3.4 & $1.40-5.4$ \\
5 & $500.1-1000$ & 24 & 1.89 & $1.50-2.29$ & 7.17 & $2.96-11.35$ \\
6 & $1000.1-2000$ & 18 & 2.36 & $1.93-2.78$ & 5.56 & $2.47-8.64$ \\
7 & $2000.1-7000$ & 22 & 3.17 & $2.66-3.68$ & 10.23 & $4.46-16.0$ \\
8 & More than 7000 & 14 & 4.09 & $2.57-5.60$ & 13.36 & $6.36-20.38$ \\
Total & & 170 & 1.87 & $1.64-2.10$ & 4.89 & $3.59-6.19$ \\
\hline
\end{tabular}

LNM = lymph node metastasis; $\mathrm{CI}=$ confidence interval.

Significant linear relationships were also observed between the preoperative serum calcitonin level and primary tumor size $\left(\mathrm{R}^{2}=0.459\right.$ and $p<0.001$, Figure S1a, supporting information) or the number of LNMs ( $\mathrm{R}^{2}=0.201$ and $p<0.001$, Figure S2a, supporting information). Subgroup analyses for sporadic MTC and hereditary MTC were also performed (Figure S1b and S2b, supporting information). When we generated plot diagrams of the mean tumor size (Figure 1a) and number of LNMs (Figure 1b) against each calcitonin group, we noticed a sharp increase in tumor size when the calcitonin level exceeded $200.1 \mathrm{pg} / \mathrm{mL}$ (the lowest limit for the 4th group), and a sharp increase in the number of LNMs when the calcitonin level exceeded $1000.1 \mathrm{pg} / \mathrm{mL}$ (the lowest limit for the sixth group).

a Tumor size and preoperative calcitonin

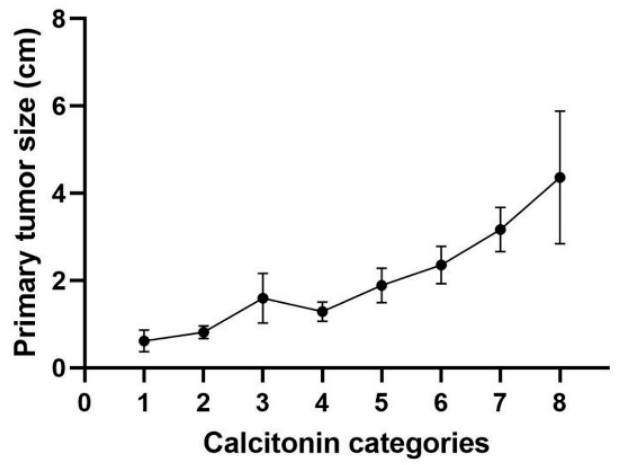

b Number of LNM and preoperative calcitonin

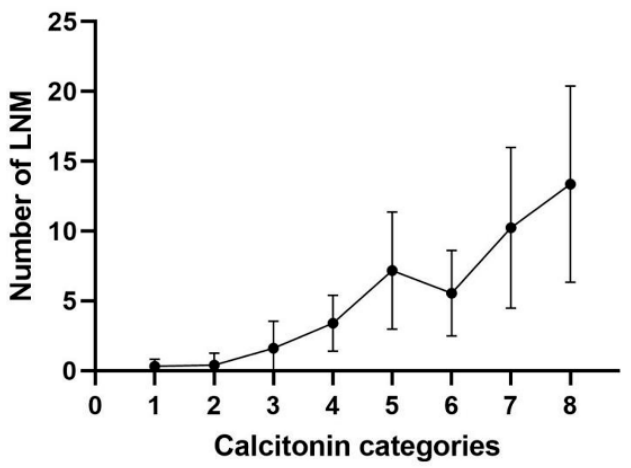

Figure 1. Correlation between tumor extent and preoperative serum calcitonin levels. (a) Size of the primary tumor. (b) Number of LNMs (Calcitonin categories: category 1 (1-20 pg/mL), category 2 (20.1-100 pg/mL), category $3(200.1-500 \mathrm{pg} / \mathrm{mL})$, category $4(200.1-500 \mathrm{pg} / \mathrm{mL})$, category $5(500.1-1000 \mathrm{pg} / \mathrm{mL})$, category 6 (1000.1-2000 pg/mL), category 7 (2000.1-7000 pg/mL) and category 8 (more than $7000 \mathrm{pg} / \mathrm{mL}$ ). The point represents the mean of the each category, and the error bars represent $95 \%$ confidential interval).

\subsection{Tumor Extent According to the Preoperative Serum Calcitonin Level}

We classified disease extent according to the region of LNM (central, ipsilateral lateral or contralateral lateral LNM) and distant metastasis. Preoperative serum calcitonin levels were significantly associated with tumor extent $(p<0.001)$. The post hoc analysis showed that ipsilateral lateral LNM, contralateral lateral LNM and distant metastasis were associated with higher calcitonin levels than no LNM or central LNM (Figure 2).

Patients with ipsilateral lateral LNM, contralateral lateral LNM and distant metastasis had preoperative serum calcitonin thresholds of 20.1, 200.1 and $500.1 \mathrm{pg} / \mathrm{mL}$, respectively. However, one patient with distant metastasis had a preoperative serum calcitonin level less than $500.1 \mathrm{pg} / \mathrm{mL}$ (109 pg/mL). Central LNM was observed in all ranges of preoperative serum calcitonin levels (Table 4). 
Initial distant metastasis was observed in eight patients, and the most common site of distant metastasis was the lung. Detailed information on these patients is provided in Table 5.

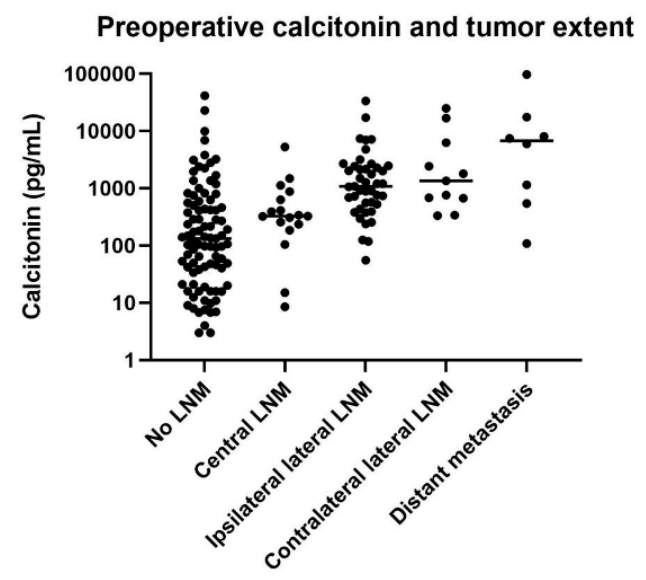

Figure 2. Correlation between tumor extent and preoperative serum calcitonin levels. The dots represent serum calcitonin level in each patient, and the horizontal lines represent the mean of the group.

Table 4. Lymph node metastasis and distant metastasis by preoperative serum calcitonin levels.

\begin{tabular}{|c|c|c|c|c|c|c|c|c|c|c|}
\hline \multirow{2}{*}{$\begin{array}{l}\text { Calcitonin } \\
\text { Category }\end{array}$} & \multirow{2}{*}{$\begin{array}{c}\text { Preoperative } \\
\text { Serum Calcitonin } \\
(\mathrm{pg} / \mathrm{mL})\end{array}$} & \multirow{2}{*}{$\begin{array}{l}\text { Number } \\
\text { of } \\
\text { Patients }\end{array}$} & \multicolumn{2}{|c|}{$\begin{array}{l}\text { Central } \\
\text { LNM }\end{array}$} & \multicolumn{2}{|c|}{$\begin{array}{c}\text { Ipsilateral } \\
\text { Lateral LNM }\end{array}$} & \multicolumn{2}{|c|}{$\begin{array}{l}\text { Contralateral } \\
\text { Lateral LNM }\end{array}$} & \multicolumn{2}{|c|}{$\begin{array}{c}\text { Distant } \\
\text { Metastasis }\end{array}$} \\
\hline & & & $n$ & $\%$ & $n$ & $\%$ & $n$ & $\%$ & $n$ & $\%$ \\
\hline 1 & $1-20$ & 22 & 2 & 9.1 & 0 & 0 & 0 & 0 & 0 & 0 \\
\hline 2 & $20.1-100$ & 22 & 1 & 4.5 & 1 & 4.5 & 0 & 0 & 0 & 0 \\
\hline 3 & $100.1-200$ & 18 & 5 & 27.8 & 3 & 16.7 & 0 & 0 & 1 & 5.6 \\
\hline 4 & $200.1-500$ & 30 & 17 & 56.7 & 9 & 30.0 & 2 & 6.7 & 0 & 0 \\
\hline 5 & 500.1-1000 & 24 & 15 & 62.5 & 14 & 58.3 & 3 & 12.5 & 1 & 4.2 \\
\hline 6 & $1000.1-2000$ & 18 & 12 & 66.7 & 10 & 55.6 & 2 & 11.1 & 1 & 5.6 \\
\hline 7 & 2000.1-7000 & 22 & 12 & 54.5 & 14 & 63.6 & 3 & 13.6 & 1 & 4.5 \\
\hline 8 & More than 7000 & 14 & 8 & 57.1 & 11 & 78.6 & 4 & 28.6 & 4 & 28.6 \\
\hline Total & & 170 & 72 & 42.4 & 62 & 36.5 & 14 & 8.2 & 8 & 4.7 \\
\hline
\end{tabular}

LNM = lymph node metastasis.

\subsection{LRs for the Preoperative Serum Calcitonin Level in Diagnosing the Extent of LNM}

We calculated the likelihood ratios (LRs) to evaluate whether the calcitonin has diagnostic value as regards the disease extent. A positive LR greater than 10.0 or a negative LR less than 0.1 can be considered statistically adequate to confirm or exclude malignancy, respectively. Additionally, a positive LR between 5 and 10, and a negative LR between 0.1 and 0.2 , are considered to have good diagnostic value. We calculated the LRs for multiple different levels of preoperative serum calcitonin (Table 6). We found that for patients with central LNM, the negative LR was 0.1 when the preoperative serum calcitonin level was less than $100 \mathrm{pg} / \mathrm{mL}$, and that for patients with ipsilateral lateral LNM, the negative LR was $0.04,0.12$ or 0.18 when the preoperative serum calcitonin level was less than 100 , 200 or $300 \mathrm{pg} / \mathrm{mL}$, respectively. For patients with contralateral lateral LNM, the negative LR was 0 when the preoperative serum calcitonin level was less than $300 \mathrm{pg} / \mathrm{mL}$ (Table 6). 
Table 5. Detailed information of patients with distant metastases.

\begin{tabular}{|c|c|c|c|c|c|c|c|c|c|c|c|}
\hline No. & $\begin{array}{l}\text { Age at } \\
\text { Dx }\end{array}$ & Sex & Sporadic/Hereditary & $\begin{array}{l}\text { Preoperative Serum } \\
\text { Calcitonin (pg/mL) }\end{array}$ & $\begin{array}{l}\text { Primary } \\
\text { Tumor } \\
\text { Size (cm) }\end{array}$ & $\begin{array}{l}\text { Number of } \\
\text { LNMs }\end{array}$ & $\begin{array}{l}\text { Central } \\
\text { LNM }\end{array}$ & $\begin{array}{l}\text { Ipsilateral } \\
\text { Lateral } \\
\text { LNM }\end{array}$ & $\begin{array}{l}\text { Contralateral } \\
\text { Lateral LNM }\end{array}$ & N Stage & $\begin{array}{l}\text { Site of the } \\
\text { Initial Distant } \\
\text { Metastasis }\end{array}$ \\
\hline 1 & 21 & M & Sporadic & 109 & 4.5 & 14 & Positive & Positive & Negative & $\mathrm{N} 1 \mathrm{~b}$ & Lung \\
\hline $2^{+}$ & 47 & M & Sporadic & 543 & 1.5 & 26 & Positive & Positive & Negative & $\mathrm{N} 1 \mathrm{~b}$ & Lung \\
\hline 3 & 39 & $\mathrm{~F}$ & Sporadic & 1150 & 0.9 & 11 & Positive & Positive & Positive & $\mathrm{N} 1 \mathrm{~b}$ & Lung \\
\hline 4 & 43 & M & Sporadic & 6012 & 2.8 & 38 & Positive & Positive & Negative & $\mathrm{N} 1 \mathrm{~b}$ & Lung \\
\hline 5 & 63 & M & Sporadic & 7488 & 7.0 & 5 & Negative & Positive & Negative & $\mathrm{N} 1 \mathrm{~b}$ & $\begin{array}{l}\text { Mediastinal soft } \\
\text { tissue }\end{array}$ \\
\hline 6 & 26 & $\mathrm{~F}$ & Sporadic & 8060 & 4.1 & 26 & Positive & Positive & Positive & N1b & Liver \\
\hline 7 & 55 & M & Sporadic & 17,450 & 2.6 & 14 & Positive & Positive & Negative & N1b & Lung, liver \\
\hline 8 & 24 & $\mathrm{~F}$ & Sporadic & 97,500 & 8.0 & 10 & Positive & Positive & Positive & N1b & Lung, bone \\
\hline
\end{tabular}


Table 6. Likelihood ratios for preoperative serum calcitonin levels in diagnosing lymph node metastasis and distant metastasis.

\begin{tabular}{|c|c|c|c|c|c|c|c|c|}
\hline $\begin{array}{l}\text { Calcitonin } \\
\text { Cut-Off } \\
(\mathrm{pg} / \mathrm{mL})\end{array}$ & $\begin{array}{c}\mathbf{N} \text { with } \\
\text { Central LNM }\end{array}$ & $\begin{array}{l}\text { N without } \\
\text { Central LNM }\end{array}$ & Positive LR & Negative LR & $\begin{array}{c}\mathbf{N} \text { with } \\
\text { Ipsilateral Lateral } \\
\text { LNM }\end{array}$ & $\begin{array}{l}\text { N without } \\
\text { Ipsilateral } \\
\text { Lateral LNM }\end{array}$ & Positive LR & Negative LR \\
\hline 20 & 2 & 20 & $1.22(1.10-1.36)$ & $0.14(0.03-0.56)$ & 0 & 22 & $1.26(1.14-1.38)$ & 0 \\
\hline 100 & 3 & 41 & $1.65(1.38-1.96)$ & $0.10(0.03-0.31)$ & 1 & 43 & $1.64(1.40-1.91)$ & $0.04(0.01-0.29)$ \\
\hline 200 & 8 & 54 & $1.98(1.57-2.50)$ & $0.20(0.10-0.40)$ & 4 & 58 & $2.02(1.63-2.50)$ & $0.12(0.05-0.32)$ \\
\hline 300 & 13 & 61 & $2.17(1.65-2.86)$ & $0.29(0.17-0.49)$ & 7 & 67 & $2.33(1.81-3.02)$ & $0.18(0.09-0.37)$ \\
\hline 400 & 23 & 62 & $1.86(1.37-2.51)$ & $0.50(0.35-0.73)$ & 12 & 73 & $2.53(1.89-3.40)$ & $0.27(0.16-0.45)$ \\
\hline 500 & 25 & 67 & $2.07(1.47-2.89)$ & $0.51(0.36-0.72)$ & 13 & 79 & $2.94(2.10-4.12)$ & $0.29(0.17-0.47)$ \\
\hline 1000 & 40 & 76 & $1.98(1.26-3.10)$ & $0.72(0.57-0.90)$ & 27 & 89 & $3.21(2.02-5.10)$ & $0.53(0.39-0.71)$ \\
\hline 3000 & 59 & 88 & $1.78(0.82-3.81)$ & $0.91(0.80-1.04)$ & 47 & 100 & $3.27(1.47-7.26)$ & $0.82(0.70-0.95)$ \\
\hline 5000 & 61 & 91 & $2.16(0.87-5.25)$ & $0.91(0.82-1.02)$ & 49 & 103 & $4.50(1.70-12.10)$ & $0.83(0.72-0.95)$ \\
\hline 7000 & 64 & 92 & $1.82(0.66-5.00)$ & $0.95(0.86-1.04)$ & 51 & 105 & $6.32(1.85-22.02)$ & $0.85(0.75-0.95)$ \\
\hline 10,000 & 68 & 94 & $1.37(0.35-5.26)$ & $0.98(0.92-1.06)$ & 56 & 106 & $5.11(1.09-25.11)$ & $0.92(0.85-1.00)$ \\
\hline Total & 72 & 98 & & & 62 & 108 & & \\
\hline $\begin{array}{l}\text { Calcitonin } \\
\text { Cut-Off } \\
(\mathrm{pg} / \mathrm{mL})\end{array}$ & $\begin{array}{c}\text { N with } \\
\text { Contralateral } \\
\text { Lateral LNM }\end{array}$ & $\begin{array}{l}\text { N without } \\
\text { Contralateral } \\
\text { Lateral LNM }\end{array}$ & Positive LR & Negative LR & $\begin{array}{c}\text { N with } \\
\text { Distant Metastasis }\end{array}$ & $\begin{array}{c}\mathrm{N} \text { without } \\
\text { Distant Metastasis }\end{array}$ & Positive LR & Negative LR \\
\hline 20 & 0 & 22 & $1.16(1.09-1.24)$ & 0 & 0 & 22 & $1.16(1.09-1.23)$ & 0 \\
\hline 300 & 0 & 74 & $1.90(1.64-2.21)$ & 0 & 1 & 73 & $1.59(1.18-2.14)$ & $0.28(0.04-1.75)$ \\
\hline 400 & 2 & 83 & $1.83(1.40-2.40)$ & $0.27(0.07-0.98)$ & 1 & 84 & $1.82(1.34-2.47)$ & $0.24(0.04-1.52)$ \\
\hline 500 & 2 & 90 & $2.03(1.53-2.69)$ & $0.25(0.07-0.90)$ & 1 & 91 & $2.00(1.46-2.74)$ & $0.22(0.04-1.40)$ \\
\hline 1000 & 5 & 111 & $2.23(1.40-3.54)$ & $0.50(0.25-1.02)$ & 2 & 114 & $2.53(1.59-4.03)$ & $0.36(0.11-1.19)$ \\
\hline 2000 & 7 & 127 & $2.69(1.45-4.99)$ & $0.61(0.36-1.04)$ & 3 & 131 & $3.27(1.75-6.09)$ & $0.46(0.19-1.14)$ \\
\hline 3000 & 8 & 139 & $3.94(1.85-8.35)$ & $0.64(0.41-1.01)$ & 3 & 144 & $5.63(2.82-11.23)$ & $0.42(0.17-1.03)$ \\
\hline 5000 & 8 & 144 & $5.57(2.47-12.57)$ & $0.62(0.39-0.98)$ & 3 & 149 & $7.81(3.69-16.46)$ & $0.41(0.17-1.00)$ \\
\hline 7000 & 10 & 146 & $4.47(1.60-12.39)$ & $0.76(0.55-1.07)$ & 4 & 152 & $8.07(3.24-20.26)$ & $0.53(0.27-1.07)$ \\
\hline 10,000 & 11 & 151 & $6.69(1.78-25.10)$ & $0.81(0.62-1.07)$ & 6 & 156 & $6.76(1.61-28.33)$ & $0.78(0.52-1.16)$ \\
\hline Total & 14 & 156 & & & 8 & 162 & & \\
\hline
\end{tabular}

$\mathrm{N}=$ number of patients; LNM = lymph node metastasis; LR = likelihood ratio; Negative LR below 0.2 and Positive LR above 5 were marked in bolds number. 


\section{Discussion}

Surgery is the only potentially curative treatment for MTC, but the initial surgical extent is controversial. Current guidelines suggest that serum calcitonin level could be considered when deciding the extent of surgery. This study found that preoperative serum calcitonin levels were closely associated with primary tumor size and the number of LNMs, and that increasing preoperative serum calcitonin was correlated with tumor extent. The thresholds of preoperative serum calcitonin according to disease extent were $20 \mathrm{pg} / \mathrm{mL}$ for ipsilateral lateral LNM, $200 \mathrm{pg} / \mathrm{mL}$ for contralateral lateral LNM, and $500 \mathrm{pg} / \mathrm{mL}$ for distant metastasis. Notably, the cut-off value for preoperative serum calcitonin with a negative LR ranging from 0.1 to 0.2 was less than $100 \mathrm{pg} / \mathrm{mL}$ in patients with central LNM and $300 \mathrm{pg} / \mathrm{mL}$ in patients with ipsilateral lateral LNM or contralateral lateral LNM. These findings indicate that the preoperative serum calcitonin level can serve as a practical biomarker to predict disease burden, namely, tumor size and number of LNMs, which is consistent with previous reports [11,13]. Furthermore, this study showed that the linear correlation between preoperative serum calcitonin category and tumor burden is significant regardless of whether the MTC was of the sporadic or hereditary type.

As biostatistical techniques have evolved over time, the use of statistical evaluation in biomarker exploration has become more important [19]. The strength of our study is that calcitonin also had diagnostic value for indicating the extent of LNM. The extent of lymph node dissection at the time of thyroidectomy is an ongoing discussion. Because the central node compartment is the site of primary lymphatic drainage from the thyroid, and LNM occurs during the early course of MTC [20,21], the guidelines of the ATA/European Thyroid Association (ETA), the British Thyroid Association (BTA), and the American Association of Endocrine Surgeons (AAES) recommend central node compartment dissection regardless of unequivocal evidence of cervical LNM $[9,16,22]$. However, the NCCN guidelines recommend total thyroidectomy with cervical dissection for primary tumors greater than $1.0 \mathrm{~cm}$ in diameter or bilateral thyroid disease [15]. Prophylactic lateral node dissection is more controversial. The preoperative serum calcitonin level and ultrasound findings can be helpful for determining the need for lateral node dissection $[9,16]$. Machens et al. reported preoperative serum calcitonin levels based on the pathologic extent of disease [17]. They suggested that preoperative serum calcitonin levels exceeding 20,50 and $200 \mathrm{pg} / \mathrm{mL}$ were associated with ipsilateral central and lateral LNM, contralateral central LNM, and contralateral lateral LNM, respectively. However, they provided only a simple threshold of the calcitonin level that depended on the extent of metastasis. Based largely on the simple threshold results of a previous study, the ATA/ETA guidelines suggest that contralateral neck dissection should be considered when the serum calcitonin level is greater than $200 \mathrm{pg} / \mathrm{mL}$. Additionally, the ATA/ETA guidelines recommend that dissection of the lateral neck compartment be determined based on serum calcitonin levels when patients have no evidence of neck metastasis on their ultrasound; however, they do not provide the exact cut-off calcitonin levels. The results from this study showed that preoperative serum calcitonin levels exceeding 20 and $200 \mathrm{pg} / \mathrm{mL}$ were associated with ipsilateral lateral LNM and contralateral lateral LNM, respectively, which is consistent with the findings of a previous study [17]. Furthermore, with our statistical analyses of patient data, we identified the preoperative serum calcitonin level that can be used as a diagnostic biomarker that reflects the disease extent. As a rule of thumb, a negative LR less than 0.1 indicates that a negative test is excellent at ruling out a diagnosis, and a negative LR less than 0.2 indicates that a negative test is good at ruling out a diagnosis $[19,23,24]$.

Most of the MTC guidelines agree that the minimal standard initial therapeutic surgical extent is total thyroidectomy with central lymph node dissection. These surgical approaches may be reasonable, but long-term results are lacking. Notably, the relationship between the extent of cervical lymph node dissection and survival advantage has not been clearly defined [25]. Furthermore, central node dissection has the potential risks of hypoparathyroidism and recurrent laryngeal nerve injury [26]. This study revealed that the negative LR for central LNM was 0.1 when the preoperative serum calcitonin level was less than $100 \mathrm{pg} / \mathrm{mL}$. Thus, consideration should be given to whether prophylactic 
central lymph node dissection should be performed in patients with a serum calcitonin level less than $100 \mathrm{pg} / \mathrm{mL}$, and with no evidence of central node metastasis on the preoperative ultrasound. Likewise, the negative LR for ipsilateral lateral LNM was less than 0.2 when the preoperative serum calcitonin level was less than $300 \mathrm{pg} / \mathrm{mL}$; therefore, prophylactic ipsilateral neck dissection in patients with no evidence of lateral node metastasis on their preoperative ultrasound also needs careful consideration. In particular, we found that the negative LR was 0 for contralateral lateral LNM when the preoperative serum calcitonin level was less than $300 \mathrm{pg} / \mathrm{mL}$. Thus, when prophylactic contralateral neck dissection is being considered in patients with documented ipsilateral lateral LNM on their preoperative ultrasound, based on our study, a preoperative serum calcitonin level of $300 \mathrm{pg} / \mathrm{mL}$ can be used as a cut-off value. Here, we calculated negative LRs according to multiple categories of calcitonin levels for use in the clinic. We still believe that the purpose of treating patients with MTC is to achieve complete surgical resection in the early stages. Despite these goals, aggressive prophylactic lymph node dissection should be considered carefully only when the need is great. Thus, experienced practitioners may use an alternative cut-off level of preoperative serum calcitonin, depending on the clinical situation.

According to the ATA, preoperative ultrasound evaluation is the most important preoperative imaging study [9]. However, the accuracy of ultrasound is highly dependent on experience and expertise [27,28]. False-negative results from preoperative ultrasounds may lead to inadequate surgical management that increases the risk of recurrence and a poor prognosis. Thus, if there is any doubt that preoperative ultrasound was performed properly, we propose that the negative LR according to the preoperative serum calcitonin level can be used to predict the extent of LNM so as to optimize the initial surgery.

This study has some limitations. One of the critical limitations of this study is that this was a retrospective design conducted in a single tertiary referral center, and accordingly selection bias might have occurred. Nonetheless, the study population included a relatively large number of patients, despite the low incidence rate of MTC. The distant metastasis group, however, is composed of a relatively small number of patients. Thus, further prospective multicenter studies are needed to validate the current results. Second, guidelines evolved during the study period. Most of the enrolled patients underwent total thyroidectomy with initial central lymph node dissection, which is consistent with current guidelines. However, 11 patients (6.5\%) out of the enrolled patients did not undergo initial central lymph node dissection. To overcome this problem, pre- and postoperative thyroid ultrasound, computer tomography (CT), postoperative serum calcitonin levels, and the clinical course for each patient were reviewed to rule out the possibility of micrometastases (supplementary Table S1). All these patients had no evidence of lymph node metastasis in their preoperative images. Of 11 patients, 1 patient was transferred out to another hospital after surgery, but this patient only had micro-MTC. Other patients had postoperative image studies done, and there was still no evidence of lymph node metastasis after thyroidectomy. Furthermore, eight patients achieved biochemical cure during the follow-up. Among the other two patients who were not biochemically cured, one of them had recurrence at the thyroid operative bed after 9 years of follow-up, and another had no evidence of disease during 8 years of follow-up. Overall, the possibility of missed micrometastases at the first surgery seems to be low. Third, in patients with advanced MTC, carcinoembryonic antigen (CEA) is considered as a useful biomarker for evaluation of disease progression, and for monitoring patients following thyroidectomy [9]. We tried to evaluate the CEA as a diagnostic biomarker biostatistically, but because of the retrospective nature of this study, we could not get enough information about the preoperative serum CEA level.

\section{Materials and Methods}

\subsection{Study Population}

In total, 246 patients who were diagnosed with MTC at the Samsung Medical Center between 1994 and 2019 were included in this study. Of these patients, 170 had preoperative serum 
calcitonin measurements available for analysis. Their clinical and pathology reports were obtained and retrospectively reviewed. This study was approved by the institutional review board of Samsung Medical Center (IRB No. 2020-07-007), and patient consent was waived in instances by the committee, because of the retrospective chart review study design and the use of only deidentified clinicopathological information.

\subsection{Analysis of Metastasis and Tumor Extent}

From the pathology reports, we obtained information on the primary tumor diameter $(\mathrm{cm})$, location and total number of LNMs, ETE, tumor extent and distant metastasis. The extent of LNM was categorized according to location: central LNM, ipsilateral lateral LNM or contralateral lateral LNM. Central LNM included both ipsilateral and contralateral LNM. Tumor extent was classified into five groups according to the presence of LNM and distant metastasis: (1) no LNM, (2) the presence of central LNM without lateral LNM or distant metastasis, (3) the presence of ipsilateral lateral LNM without contralateral lateral LNM or distant metastasis (regardless of the central LNM status), (4) the presence of contralateral lateral LNM without distant metastasis (regardless of the central LNM status), and (5) the presence of distant metastasis (regardless of LNM). Initial distant metastasis was defined as distant metastases that were detected within 6 months after the initial surgery. Distant metastatic lesions were detected by chest and/or abdominopelvic CT, magnetic resonance imaging (MRI), whole-body bone scintigraphy, and 19-fluorodeoxyglucose positron emission tomography (FDG-PET), and/or were pathologically confirmed. For anatomical staging, the 8th edition of the American Joint Cancer Committee (AJCC) system was used.

\subsection{Measurement of Serum Calcitonin}

The serum calcitonin concentration was measured with a two-site immunoradiometric assay using a commercial kit (MEDGENIX CT-U.S.-IRMA kit, BioSource Europe S.A., Belgium). The minimum detectable limit was $0.9 \mathrm{pg} / \mathrm{mL}$. All samples were measured in duplicate, and the intraassay and interassay coefficients of variation were $2.4-3.4 \%$ and $3.6-5.4 \%$, respectively.

Preoperative serum calcitonin was divided into eight categories considering the number of patients and previous reported calcitonin threshold according to disease extent [17]: category 1 (1-20 pg/mL), category $2(20.1-100 \mathrm{pg} / \mathrm{mL})$, category $3(100.1-200 \mathrm{pg} / \mathrm{mL})$, category $4(200.1-500 \mathrm{pg} / \mathrm{mL})$, category 5 (500.1-1000 pg/mL), category 6 (1000.1-2000 pg/mL), category 7 (2000.1-7000 pg/mL), and category 8 (more than $7000 \mathrm{pg} / \mathrm{mL}$ ).

\subsection{Statistical Analysis}

Descriptive statistics were analyzed using Student's t-test or the Kruskal-Wallis test as appropriate, and are presented as means \pm standard deviations (SDs) or medians and interquartile ranges (IQRs). One-way ANOVA or the Mann-Whitney U test with Bonferroni's method were performed for the post hoc analysis of descriptive statistics. Categorical variables were analyzed using the chi-square test or Fisher's exact test and are presented as absolute numbers (percentages). Spearman correlation analysis was used to investigate the strength of an association between two continuous variables, and is represented by the correlation coefficient, rho. Linear regression analysis was performed to assess variables that could be associated with the serum calcitonin elevation in univariable and multivariable analysis. To assess continuous serum calcitonin levels, transformed calcitonin with common log (log-calcitonin) was used due to non-normality in the linear regression analysis. Positive and negative LRs were calculated using an established method [19,23]. A $p$ value $<0.05$ was considered to indicate statistical significance. Statistical analysis was performed using SPSS version 25.0 for Windows (IBM, Chicago, IL, USA). 


\section{Conclusions}

The preoperative serum calcitonin level correlated with disease extent and showed excellent diagnostic value for predicting the concrete extent of LNM. Our results suggest that preoperative serum calcitonin levels can be used to determine the optimal initial surgical extent to reduce the number of reoperations while minimizing surgical complications.

Supplementary Materials: The following are available online at http://www.mdpi.com/2072-6694/12/10/2894/s1, Figure S1: Correlation between log-transformed preoperative calcitonin level and primary tumor size; Figure S2: Correlation between preoperative log-calcitonin and the number of LNMs; Table S1: Clinical course and pre- and postoperative calcitonin and images in 11 patients who did not undergo central lymph node dissection

Author Contributions: Conceptualization, H.P. and T.H.K.; Data curation, H.P., J.P., M.S.C., J.K. and T.H.K.; Formal analysis, H.P. and T.H.K.; Investigation, H.K., J.H.S., J.-H.K., J.S.K., S.W.K. and J.H.C.; Writing-original draft, H.P.; Writing—review and editing, H.K., J.H.S., J.-H.K., J.S.K., S.W.K., J.H.C. and T.H.K. All authors have read and agreed to the published version of the manuscript.

Funding: This research was supported by a CRP-achievement grant (OTA1810531) from Samsung Medical Center.

Conflicts of Interest: The authors declare no conflict of interest.

\section{References}

1. Noone, A.M.; Cronin, K.A.; Altekruse, S.F.; Howlader, N.; Lewis, D.R.; Petkov, V.I.; Penberthy, L. Cancer Incidence and Survival Trends by Subtype Using Data from the Surveillance Epidemiology and End Results Program, 1992-2013. Cancer Epidemiol. Biomark. Prev. 2017, 26, 632-641. [CrossRef] [PubMed]

2. Utiger, R.D. Medullary thyroid carcinoma, genes, and the prevention of cancer. N. Engl. J. Med. 1994, 331, 870-871. [CrossRef]

3. Cho, B.Y.; Choi, H.S.; Park, Y.J.; Lim, J.A.; Ahn, H.Y.; Lee, E.K.; Kim, K.W.; Yi, K.H.; Chung, J.K.; Youn, Y.K.; et al. Changes in the clinicopathological characteristics and outcomes of thyroid cancer in Korea over the past four decades. Thyroid 2013, 23, 797-804. [CrossRef] [PubMed]

4. Grozinsky-Glasberg, S.; Benbassat, C.A.; Tsvetov, G.; Feinmesser, R.; Peretz, H.; Shimon, I.; Lapidot, M. Medullary thyroid cancer: A retrospective analysis of a cohort treated at a single tertiary care center between 1970 and 2005. Thyroid 2007, 17, 549-556. [CrossRef]

5. Roman, S.; Lin, R.; Sosa, J.A. Prognosis of medullary thyroid carcinoma: Demographic, clinical, and pathologic predictors of survival in 1252 cases. Cancer 2006, 107, 2134-2142. [CrossRef]

6. Sebastian, S.O.; Gonzalez, J.M.; Paricio, P.P.; Perez, J.S.; Flores, D.P.; Madrona, A.P.; Romero, P.R.; Tebar, F.J. Papillary thyroid carcinoma: Prognostic index for survival including the histological variety. Arch. Surg. 2000, 135, 272-277. [CrossRef]

7. Costante, G.; Durante, C.; Francis, Z.; Schlumberger, M.; Filetti, S. Determination of calcitonin levels in C-cell disease: Clinical interest and potential pitfalls. Nat. Clin. Pract. Endocrinol. Metab. 2009, 5, 35-44. [CrossRef]

8. Karges, W. Calcitonin determination for early diagnosis of medullary thyroid cancer. Chirurg 2010, 81, $622-626$.

9. Wells, S.A., Jr.; Asa, S.L.; Dralle, H.; Elisei, R.; Evans, D.B.; Gagel, R.F.; Lee, N.; Machens, A.; Moley, J.F.; Pacini, F.; et al. Revised American Thyroid Association guidelines for the management of medullary thyroid carcinoma. Thyroid 2015, 25, 567-610. [CrossRef]

10. Miyauchi, A.; Matsuzuka, F.; Kuma, K.; Takai, S.; Nakamoto, K.; Nakamura, K.; Nanjo, S.; Maeda, M. Evaluation of surgical results and prediction of prognosis in patients with medullary thyroid carcinoma by analysis of serum calcitonin levels. World J. Surg. 1988, 12, 610-615. [CrossRef]

11. Yip, D.T.; Hassan, M.; Pazaitou-Panayiotou, K.; Ruan, D.T.; Gawande, A.A.; Gaz, R.D.; Moore, F.D., Jr.; Hodin, R.A.; Stephen, A.E.; Sadow, P.M.; et al. Preoperative basal calcitonin and tumor stage correlate with postoperative calcitonin normalization in patients undergoing initial surgical management of medullary thyroid carcinoma. Surgery 2011, 150, 1168-1177. [CrossRef]

12. Machens, A.; Schneyer, U.; Holzhausen, H.J.; Dralle, H. Prospects of remission in medullary thyroid carcinoma according to basal calcitonin level. J. Clin. Endocrinol. Metab. 2005, 90, 2029-2034. [CrossRef] 
13. Cohen, R.; Campos, J.M.; Salaun, C.; Heshmati, H.M.; Kraimps, J.L.; Proye, C.; Sarfati, E.; Henry, J.F.; Niccoli-Sire, P.; Modigliani, E. Preoperative calcitonin levels are predictive of tumor size and postoperative calcitonin normalization in medullary thyroid carcinoma. Groupe d'Etudes des Tumeurs a Calcitonine (GETC). J. Clin. Endocrinol. Metab. 2000, 85, 919-922. [CrossRef]

14. Cho, Y.Y.; Jang, H.W.; Jang, J.Y.; Kim, T.H.; Choe, J.H.; Kim, J.H.; Kim, J.S.; Kim, S.W.; Chung, J.H. Clinical outcomes of patients with hypercalcitoninemia after initial treatment for medullary thyroid cancer and postoperative serum calcitonin cutoffs for predicting structural recurrence. Head Neck 2016, 38, 1501-1508. [CrossRef]

15. Haddad, R.I.; Nasr, C.; Bischoff, L.; Busaidy, N.L.; Byrd, D.; Callender, G.; Dickson, P.; Duh, Q.Y.; Ehya, H.; Goldner, W.; et al. NCCN Guidelines Insights: Thyroid Carcinoma, Version 2.2018. J. Natl. Compr. Cancer Netw. 2018, 16, 1429-1440. [CrossRef]

16. Patel, K.N.; Yip, L.; Lubitz, C.C.; Grubbs, E.G.; Miller, B.S.; Shen, W.; Angelos, P.; Chen, H.; Doherty, G.M.; Fahey, T.J., 3rd; et al. The American Association of Endocrine Surgeons Guidelines for the Definitive Surgical Management of Thyroid Disease in Adults. Ann. Surg. 2020, 271, e21-e93. [CrossRef]

17. Machens, A.; Dralle, H. Biomarker-based risk stratification for previously untreated medullary thyroid cancer. J. Clin. Endocrinol. Metab. 2010, 95, 2655-2663. [CrossRef]

18. Rohmer, V.; Vidal-Trecan, G.; Bourdelot, A.; Niccoli, P.; Murat, A.; Wemeau, J.L.; Borson-Chazot, F.; Schvartz, C.; Tabarin, A.; Chabre, O.; et al. Prognostic factors of disease-free survival after thyroidectomy in 170 young patients with a RET germline mutation: A multicenter study of the Groupe Francais d'Etude des Tumeurs Endocrines. J. Clin. Endocrinol. Metab. 2011, 96, E509-E518. [CrossRef]

19. Ray, P.; Le Manach, Y.; Riou, B.; Houle, T.T. Statistical evaluation of a biomarker. Anesthesiology 2010, 112, 1023-1040. [CrossRef]

20. Moley, J.F.; DeBenedetti, M.K. Patterns of nodal metastases in palpable medullary thyroid carcinoma: Recommendations for extent of node dissection. Ann. Surg. 1999, 229, 880-888. [CrossRef]

21. Scollo, C.; Baudin, E.; Travagli, J.P.; Caillou, B.; Bellon, N.; Leboulleux, S.; Schlumberger, M. Rationale for central and bilateral lymph node dissection in sporadic and hereditary medullary thyroid cancer. J. Clin. Endocrinol. Metab. 2003, 88, 2070-2075. [CrossRef] [PubMed]

22. Perros, P.; Boelaert, K.; Colley, S.; Evans, C.; Evans, R.M.; Gerrard Ba, G.; Gilbert, J.; Harrison, B.; Johnson, S.J.; Giles, T.E.; et al. Guidelines for the management of thyroid cancer. Clin. Endocrinol. (Oxf.) 2014, 81 (Suppl. 1), 1-122. [CrossRef] [PubMed]

23. Grimes, D.A.; Schulz, K.F. Refining clinical diagnosis with likelihood ratios. Lancet 2005, 365, 1500-1505. [CrossRef]

24. Altman, D.G.; Bland, J.M. Diagnostic tests 2: Predictive values. BMJ 1994, 309, 102. [CrossRef]

25. Kandil, E.; Gilson, M.M.; Alabbas, H.H.; Tufaro, A.P.; Dackiw, A.; Tufano, R.P. Survival implications of cervical lymphadenectomy in patients with medullary thyroid cancer. Ann. Surg. Oncol. 2011, 18, 1028-1034. [CrossRef] [PubMed]

26. Wang, T.S.; Cheung, K.; Farrokhyar, F.; Roman, S.A.; Sosa, J.A. A meta-analysis of the effect of prophylactic central compartment neck dissection on locoregional recurrence rates in patients with papillary thyroid cancer. Ann. Surg. Oncol. 2013, 20, 3477-3483. [CrossRef]

27. Oltmann, S.C.; Schneider, D.F.; Chen, H.; Sippel, R.S. All thyroid ultrasound evaluations are not equal: Sonographers specialized in thyroid cancer correctly label clinical N0 disease in well differentiated thyroid cancer. Ann. Surg. Oncol. 2015, 22, 422-428. [CrossRef]

28. Kumbhar, S.S.; O’Malley, R.B.; Robinson, T.J.; Maximin, S.; Lalwani, N.; Byrd, D.R.; Wang, C.L. Why Thyroid Surgeons Are Frustrated with Radiologists: Lessons Learned from Pre- and Postoperative US. Radiographics 2016, 36, 2141-2153. [CrossRef]

Publisher's Note: MDPI stays neutral with regard to jurisdictional claims in published maps and institutional affiliations. 